\title{
UM LEVANTAMENTO QUANTITATIVO DA UTILIZAÇÃO DO INSTRUMENTO KIDSCREEN NA AVALIAÇÃO DA QUALIDADE DE VIDA DE CRIANÇAS: UMA REVISÃO NA PRODUÇÃO CIENTÍFICA UTILIZANDO A BASE DE DADOS SCOPUS
}

\author{
A QUANTITATIVE SURVEY OF THE USE OF THE KIDSCREEN \\ INSTRUMENT IN THE QUALITY ASSESSMENT OF CHILDREN: A REVIEW \\ OF SCIENTIFIC PRODUCTION USING THE SCOPUS DATABASE
}

\section{Mylena Aparecida Rodrigues Alves ${ }^{1}$}

1 Mylena Aparecida Rodrigues Alves. Mestranda pelo Programa de Pós-Graduação em Ciências da Saúde pela Universidade Estadual de Ponta Grossa (UEPG), Ponta Grossa, PR, Brasil. E-mail: mylena_cg@hotmail.com.

\section{Guilherme Moreira Caetano Pinto $^{2}$}

2 Guilherme Moreira Caetano Pinto. Mestrando em Engenharia de Produção pela Universidade Tecnológica Federal do Paraná (UTFPR), Ponta Grossa, PR, Brasil. E-mail: guilherme-coxa@ uol.com.br

\section{Márcia Helena Baldani Pinto $^{3}$}

3 Márcia Helena Baldani Pinto. Doutora em Saúde Pública pela Universidade de São Paulo (USP), São Paulo, SP, Brasil. E-mail:mbaldani@uepg.br

\section{Bruno Pedroso 4}

4 Bruno Pedroso. Doutor em Educação Física pela Universidade Estadual de Campinas (UNICAMP), SP, Brasil. E-mail: prof.brunopedroso@gmail.com

\section{RESUMO}

O objetivo do presente foi realizar um levantamento quantitativo bibliográfico sobre a utilização dos instrumentos KIDSCREEN na literatura acadêmica. Para tal, utilizaram-se as palavras-chave "Quality of Life" e "KIDSCREEN" para a busca na base de dados da SCOPUS, o espaço temporal selecionado considerou todas as publicações anteriores até o ano de 2016. O retorno foi de 317 artigos científicos. Destes, 148 foram excluídos por não se enquadrarem nos critérios de inclusão e, consequentemente, foram selecionados 169 artigos para análise na presente pesquisa. Os periódicos que obtiveram mais publicações foram o Quality of Life Research, e o Health and Quality of Life Outcomes. Em relação à utilização das três versões do instrumento $\operatorname{KIDSCREEN~(52,~} 27$ e 10), a versão 52 foi a mais utilizada pelos pesquisadores, seguida pela versão 27 . A aplicação do instrumento KIDSCREEN ocorreu em maior frequência por autores da Europa (Alemanha e Espanha) e da América do Sul (Brasil e do Chile), totalizando 29\% do total de publicações selecionadas para a presente pesquisa. Concluiu-se que o uso dos instrumentos KIDSCREEN vem crescendo nos últimos anos e são usados em diversos grupos, de populações gerais e específicas, o que sugere sua eficiência em ambos os casos.

PALAVRAS-CHAVE: KIDSCREEN. Qualidade de vida. Avaliação. Crianças. Adolescentes. 


\section{ABSTRACT}

The goal of the present study was to carry out a quantitative bibliographic survey on the use of KIDSCREEN instruments in the academic literature, by searching the SCOPUS database. To do this, the keywords "Quality of Life" and "KIDSCREEN" were used to search the SCOPUS database, the selected timeframe considered all previous publications up to the year 2016. The return was 317 scientific articles. Of these, 148 were excluded because they did not fit the inclusion criteria and, consequently, 169 articles were selected for analysis in the present research. The most frequently used journals were the Quality of Life Research, and the Health and Quality of Life Outcomes. In relation to the use of the three versions of the KIDSCREEN instrument (52, 27 and 10), version 52 was the most used by the researchers, followed by version 27 . The application of the KIDSCREEN instrument was most frequently performed by authors from Europe (Germany and Spain) and South America (Brazil and Chile), totaling 29\% of the total number of publications selected for the present study. It was concluded that the KIDSCREEN instruments have been growing in recent years and are used in several groups of general and specific populations, which suggests their efficiency in both cases.

KEYWORDS: KIDSCREEN. Quality of life. Evaluation. Children. Adolescents.

\section{INTRODUÇÃO}

Conceitualmente o termo qualidade de vida, em virtude de sua complexidade, não se delimita a um conceito único. Na década de 90 , com objetivo de reduzir os equívocos acerca de sua conceituação, as discussões sobre o conceito da qualidade de vida se acentuaram (SOARES et al., 2011). Neste contexto, o Grupo de qualidade de vida da Organização Mundial da Saúde - WHOQOL - definiu o termo qualidade de vida como "a percepção do indivíduo de sua posição na vida, no contexto da cultura e sistema de valores nos quais ele vive e em relação aos seus objetivos, expectativas, padrões e preocupações" (FLECK, 2008).

De maneira geral, a entrevista e a aplicação de questionários são as metodologias mais comuns encontradas na literatura para avaliar a qualidade de vida nos mais diferentes grupos populacionais. Pondera-se que, segundo Seidl e Zannon (2004), não há diferenças significativas nos resultados obtidos na avaliação de qualidade de vida quando empregadas essas duas formas de pesquisa.

Neste sentido, para que fosse possível a avaliação da qualidade de vida o grupo WHOQOL disponibilizou alguns instrumentos voltados a este fim. Entre os instrumentos de avaliação da qualidade de vida produzidos destacam-se o WHOQOL-100 e WHOQOL-bref, voltados à população geral. Destes, considerados progenitores, surgiram diversos outros instrumentos que avaliam a qualidade de vida em populações com condições específicas, como o WHOQOL-DIS, WHOQOL-HIV, WHOQOL-OLD e WHOQOL-Pain.

O procedimento de avaliação da qualidade de vida em crianças se justifica na premissa de que problemas relacionados com a qualidade de vida durante a infância podem repercutir na adolescência e idade adulta e, desta forma, a avaliação pode vir a 
se tornar um meio de se reduzir tais imbróglios (JIROJANAKUL; SKEVINGTON, 2000).

Ressalta-se ainda, neste sentido, que o número de instrumentos disponíveis para a avaliação da qualidade de vida em crianças e adolescente aumentou em nível mundial, tendo em vista o surgimento de 30 instrumentos genéricos e 64 instrumentos específicos direcionados a este público até o ano de 2008 (RAVENS-SIEBER et al., 2004).

Dentre os questionários de avaliação da qualidade de vida direcionados ao público infantil destacam-se os instrumentos KIDSCREEN. Estes foram elaborados pelo grupo KIDSCREEN para uso na epidemiologia em saúde pública, estudos de intervenção clínica e projetos de pesquisa5. Pesam em favor dos instrumentos KIDSCREEN o fato de que seu desenvolvimento ocorreu de forma transcultural em 13 países europeus e seguiu um rigoroso, claro e bem sistematizado procedimento metodológico. Além disso, seu constructo detém propriedades psicométricas satisfatórias e o projeto final de construção resultou em três versões: KIDSCREEN-52, KIDSCREEN-27 e KIDSCREEN-10 (HIDALGO-RAMUSSEN et al., 2014; GASPAR; MATOS, 2008).

O KIDSCREEN-52 é a versão progenitora, tem característica genérica e contém 10 dimensões, sendo elas a Saúde e Atividade Física, Sentimentos, Estado Emocional, Auto-percepção, Autonomia e Tempo Livre, Família/Ambiente Familiar, Aspecto Financeiro, Amigo e Apoio Social, Ambiente Escolar e Provocações/Bullying. Já o KIDSCREEN-27 (versão curta), também de característica genérica e contém cinco dimensões, entre elas o Bem-estar Físico, Bem-estar Psicológico, Autonomia, Suporte Social e Grupo de Pares e Ambiente Escolar. Por fim, o KIDSCREEN-10 contém dez questões, tem característica unidimensional e apresenta um escore global da qualidade de vida.

Os instrumentos KIDSCREEN, vêm sendo utilizado em diversas pesquisas ao redor do mundo. Além disso, tendo em vista sua disponibilização em três versões, há a possibilidade de que o KIDSCREEN seja aplicado em pesquisas com diferentes objetivos, podendo ocorrer distorções em sua utilização por pesquisadores da área.

Sendo assim, visando elucidar em que contexto o KIDSCREEN vem sendo aplicado no meio acadêmico e, desta forma, facilitar a tomada de decisão na escolha de qual instrumento para avaliar a qualidade de vida de crianças e adolescentes deve ser utilizado, bem como na perspectiva de enriquecer o meio acadêmico com conhecimentos sobre os instrumentos KIDSCREEN, a presente pesquisa tem por objetivo realizar um levantamento bibliográfico sobre a utilização dos instrumentos KIDSCREEN através da base SCOPUS em pesquisas acadêmicas em diversos países e continentes.

\section{METODOLOGIA}

A presente pesquisa foi delineada conforme a classificação de Gil (2002), em que se caracteriza como: pesquisa Exploratória em relação aos objetivos, diante da perspectiva de um problema ainda não solucionado; trata-se de uma pesquisa bibliográfica, quanto aos procedimentos metodológicos; e, por fim, caracteriza-se como um estudo quantitativo em relação à análise de dados, diante da utilização de cálculos estatísticos descritivos e interpretações de gráficos e tabelas.

A base de dados utilizada para a busca na literatura científica e técnica foi a SCOPUS. A base SCOPUS é considerada a maior base de dados de resumos e referências do mundo, lançada pela editora Elsevier, em 2004, tendo dados Bibliográficos da Biblioteca Nacional de Medicina dos Estados Unidos e contém mais de 18 milhões de referências a artigos de jornais científicos (GRÁCIO; OLIVEIRA, 2012).

Para a coleta dos artigos, utilizou-se as palavras-chave "Quality of Life" e "KIDSCREEN", direcionando a busca em "todos os títulos dos artigos, resumos e pala- 
vras-chaves". O espaço temporal selecionado considerou todas as publicações anteriores até o ano de 2016.

Posteriormente, a fim de analisar estudos acadêmicos que atendem aos objetivos deste estudo, foram adotados os seguintes critérios de inclusão: ser um estudo disponível na íntegra, estar publicado até o ano de 2016, ter como instrumento principal o KIDSCREEN em sua metodologia, não possuir estudos de validade do instrumento e não conter estudos replicados em uma mesma busca.

A análise dos artigos retornados ocorreu por meio do levantamento dos seguintes indicadores: o ano de publicação; o público pelo qual foi estudado; país e continente do primeiro autor; nome do periódico e o instrumento o qual foi utilizado (KIDSCREEN-10, KIDSCREEN-27 OU KIDSCREEN-52), totalizando assim cinco variáveis analisadas.

Os dados retornados foram planificados por meio do software Microsoft Excel 2013 e, posteriormente, analisados por meio de um fluxograma e estatísticas descritivas.

\section{RESULTADOS E DISCUSSÕES}

Inicialmente, a busca na base SCOPUS retornou 317 artigos científicos. Destes, 148 foram excluídos por não se enquadrarem nos critérios de inclusão e, consequentemente, foram selecionados 169 artigos para análise na presente pesquisa. O fluxograma 1, mostra os resultados obtidos pela presente busca.

Fluxograma 1: Desenho da busca feita na base de dados SCOPUS sobre a avaliação da qualidade de vida por meio dos instrumentos KIDSCREEN

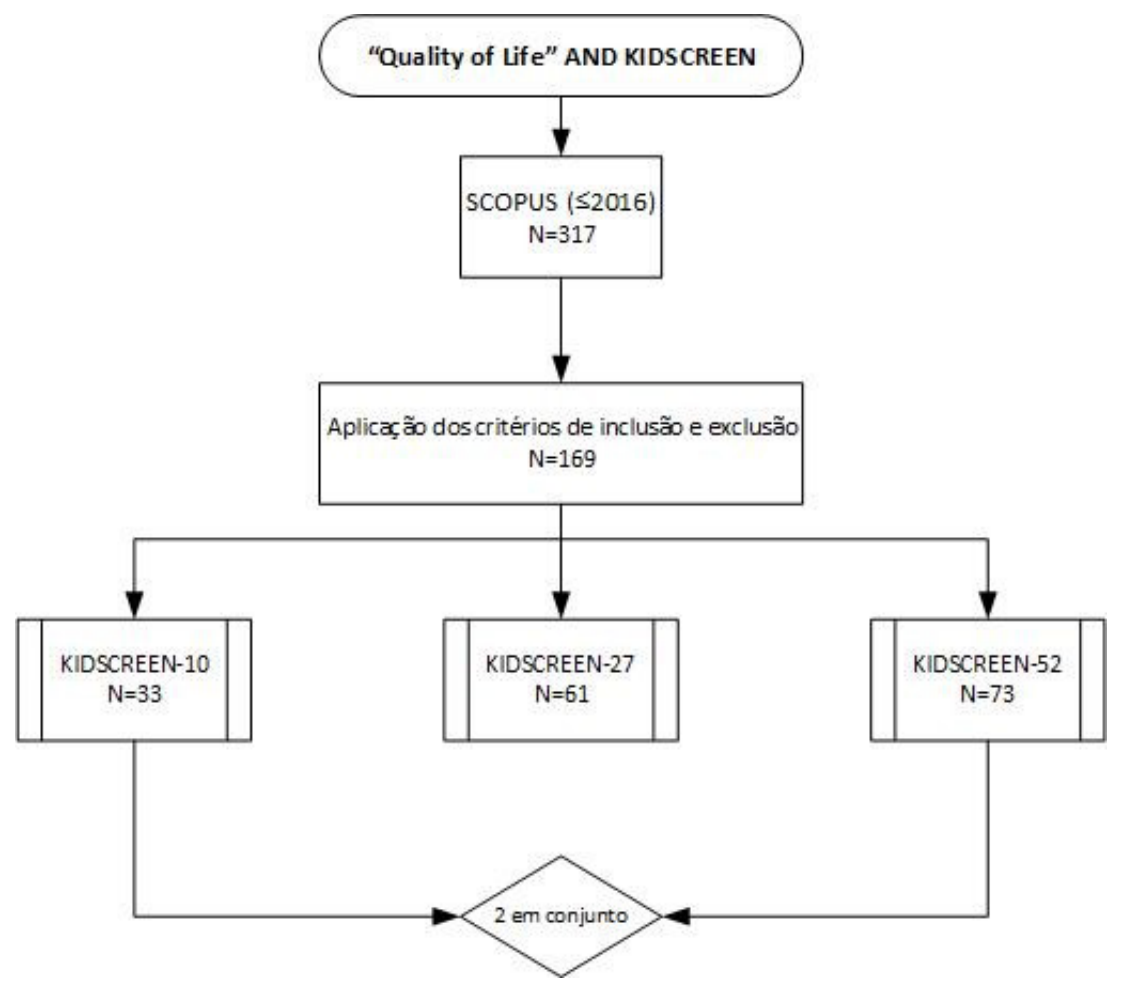

Fonte: Autoria própria 
Nota-se que o instrumento KIDSCREEN-52 (73) demonstrou um maior número de ocorrências. Tal cenário justifica-se em virtude de o KIDSCREEN-52 ser o instrumento progenitor e, dentre seus pares, apresenta uma maior multidimensionalidade em relação às outras versões. O qual, também, justifica o fato de que o instrumento KIDSCREEN-27 (61) ter sido o segundo mais utilizado.

E, por fim, a pouca utilização do instrumento KIDSCREEN-10, que embora leve um menor tempo de preenchimento ocorreu em apenas $20,7 \%$ dos trabalhos analisados. Dessa maneira, totalizou 169 artigos que utilizaram como instrumento principal, para avaliar a qualidade de vida de crianças e adolescentes, alguma versão do KIDSCREEN. Já o gráfico 1, mostra a distribuição de publicação por ano.

Gráfico 1: publicações cientificas por ano sobre a utilização dos instrumentos KIDSCREEN

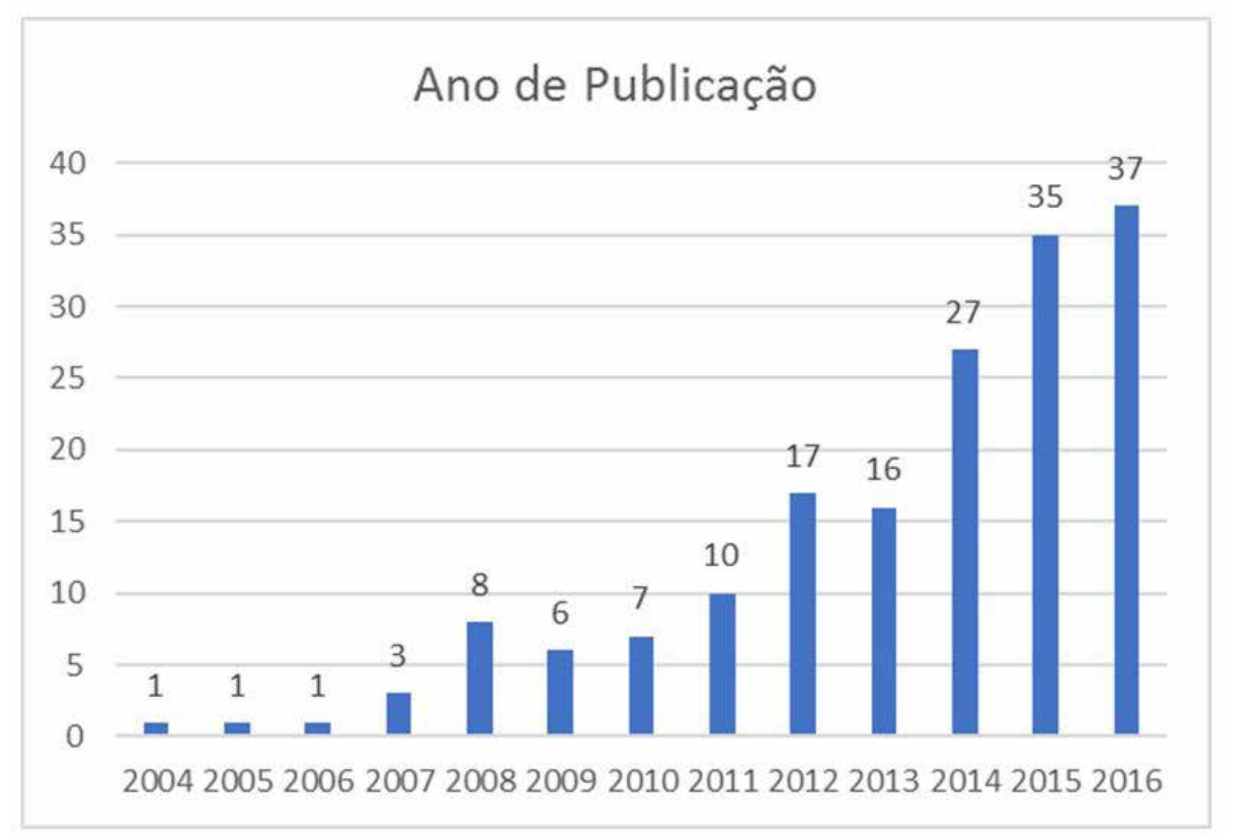

Fonte: Autoria própria (2016)

Os anos de 2001, 2002 e 2003 não foram incluídos nessa busca, pois se tratavam exclusivamente da construção do instrumento KIDSCREEN e não a utilização do mesmo.

Verifica-se que no ano de 2014 em diante houve um aumento do número de pesquisas que utilizaram o KIDSCREEN como instrumento de análise da qualidade de vida em crianças e adolescentes. Além disso, observa-se que no ano de 2014 houve um aumento no número de pesquisas com o instrumento KIDSCREEN de 8,33\% (11) em relação ao ano anterior, configurando assim o maior aumento.

Neste sentido, o estudo de Soares et al. (2011) foi arrolado à presente discussão, em virtude de suas considerações em relação a avaliação da qualidade de vida em crianças e adolescentes. $O$ autor afirma que até o ano de publicação do seu estudo (2011), as pesquisas relacionadas à qualidade de vida de crianças e adolescentes no Brasil ainda eram escassas, possivelmente em razão da pouca disponibilidade de instrumentos com este fim.

Ressalta-se ainda, quanto ao crescimento no número de pesquisas com o in- 
strumento KIDSCREEN ao redor do mundo, que este panorama reforça os resultados encontrados por Landeiro et al. (2011), que apresenta um aumento efetivo de publicações sobre a qualidade de vida em diversas populações ao longo dos anos.

Em relação aos periódicos com o maior número de publicação, a tabela 1 mostrará os dois melhores ranqueados com base na presente busca, no período de 2001 a 2015 (Quality of Life Research e o Health And Quality), e os dois melhores ranqueados (Health And Quality e o PLOS ONE) no ano de 2016.

Tabela 1: periódicos com maior número de publicações

\begin{tabular}{lccccc}
\hline \multicolumn{1}{c}{ REVISTA } & JCR & Qualis & $\mathbf{2 0 0 1 -}$ & $\mathbf{2 0 1 6}$ & No total \\
\hline QUALITY OF LIFE RESEARCH & 2,344 & A2 & 14 & 0 & 14 \\
HEALTH AND QUALITY OF LIFE OUTCOMES & 6,426 & A2 & 11 & 2 & 13 \\
PLOS ONE & 2,806 & A1 & 0 & 3 & 3 \\
\hline
\end{tabular}

Qualis: Sistema brasileiro de classificação dos periódicos (2017)

Nota se que os periódicos Quality of Life Research e o Health And Quality of Life Outcomes estão em destaque, com 14 e 13 artigos publicados, respectivamente, os quais juntos obtiveram $15,9 \%$ do total das publicações. Em sequência, o periódico PLoS ONE obteve três publicações no total. Salienta que o periódico PLoS ONE no ano de 2016 foi o que mais publicou, obtendo 8,1\% das publicações referente ao ano de 2016. Com base nisso, o periódico PLoS ONE pode ser caracterizado em ascensão em relação as publicações relacionadas sobre o instrumento KIDSCREEN.

Tal cenário explica-se devido os dois melhores ranqueados apresentarem em seu escopo uma íntima relação com a qualidade de vida. No que concerne à revista Quality of Life Research, seu escopo refere-se "a qualidade de vida relacionada à saúde, com ênfase em intervenções médicas e psicossociais". Em medida semelhante, o escopo do periódico Health and Quality of Life Outcomes é "qualidade de vida e aplicações nas ciências biológicas e sociais".

Não obstante Correia e De Carlo (2012), em sua revisão em busca de periódicos indexados sobre a qualidade de vida no contexto dos cuidados paliativo, apresentou as revistas Quality of Life Research e Health and Quality of Life Outcomes como os periódicos mais utilizados. Tal cenário coaduna o cenário encontrado pelo presente estudo, reforçando a tendência de que pesquisas relacionadas à temática da qualidade de vida busquem submissões nestes periódicos.

No que concerne aos dados retornados na análise por características do público investigado (tabela 2) foram nomeados 13 grupos, sendo eles: População geral, doenças crônicas, outros, paralisia cerebral, saúde mental, sobrepeso/obesidade, anomalia congênita, deficiência física, doenças respiratórias, violência (psicológica, sexual e física), epilepsia, dores lombares e doenças inflamatórias intestinais.

Vale explicitar que a população geral engloba estudantes, participantes de projetos e atletas, ou seja, indivíduos caracterizados sem algum tipo de anormalidade na saúde e no ambiente social. As Doenças Crônicas englobaram em sua grande parte indivíduos com câncer e diabetes. Foi classificado como saúde mental, todos os acontecimentos da saúde mental e os transtornos mentais (estresse, autismo e o transtorno de déficit de atenção). O grupo designado Violência, englobou os trabalhos que descreveram em sua metodologia os abusos sexuais, maus-tratos e bullying em crianças e adolescentes.

No grupo "outros", caracterizado por doenças altamente especificas, por se tratarem de trabalhos com uma única ocorrência, foram compostas por doenças agudas, doenças raras, transplantes, implantes, esferocitose hereditária, doença celíaca e doença falciforme. 
Tabela 2: público alvo em relação aos anos publicados

\section{GRUPO} 2001-2015 2016 Total

\begin{tabular}{lccc}
\hline 1. População geral & 53 & 13 & 66 \\
2. Doenças Crônicas & 15 & 3 & 18 \\
3. Paralisia Cerebral & 10 & 3 & 13 \\
4. Saúde Mental & 10 & 3 & 13 \\
5. Sobrepeso/obesidade & 7 & 1 & 8 \\
6. Anomalia Congênita & 5 & 2 & 7 \\
7. Deficiência Física & 4 & - & 4 \\
8. Doenças Respiratórias & 4 & - & 4 \\
9. Violência (psicológica, sexual, física) & 3 & - & 3 \\
10. Epilepsia & 3 & - & 3 \\
11. Dores lombares & 3 & - & 3 \\
12. Doenças Inflamatórias Intestinais & - & 4 & 4 \\
13. Outros & 15 & 8 & 23 \\
\hline
\end{tabular}

Fonte: Autoria própria

Nota-se que o instrumento KIDSCREEN vem sendo utilizado para avaliar a qualidade de vida no público infanto-juvenil em públicos com características de vida diversas. Ressalta-se que o rigor metodológico e a alta confiabilidade dos instrumentos KIDSCREEN, a intenção de que este instrumento descrevesse determinantes da saúde física e mental do público infanto-juvenil, e sua disponibilização para a utilização por diversos profissionais (da saúde pública, psicologia da saúde, epidemiologia, medicina e pesquisa/investigação clínica) e em ambientes distintos (hospitais, estabelecimentos médicos, escolas) justificam este cenário (GASPAR; MATOS, 2008; GASPAR, 2006).

Verifica-se também a existência de muitos trabalhos envolvendo as versões KIDSCREEN no grupo da população geral. Isto tende a limitar futuras pesquisas com este público, tendo em vista que quanto maior é a exploração em um público, menor são as chances de que os estudos tornem-se inovadores.

Grupos como a Paralisia Cerebral (13), Doenças Crônicas (18), Epilepsia (3) e Anomalia Congênita (7), foram avaliados pelo instrumento KIDSCREEN, ainda que estes encontrem-se em condições de vida específicas. Porém, Jervaeus, Kottorp e Wettergren (2013), mostram que o instrumento KIDSCREEN-52 funciona bem em crianças com câncer e diabetes em tratamento, mas deve-se ter o cuidado com o número da amostra e algumas questões unidimensionais contidas no instrumento, devido a especificidade encontrada em cada caso.

Ao que parece devido à característica do instrumento KIDSCREEN em abordar a QVRS, este vem sendo aplicado em grupos que vivem em condição de vida específica. Porém, vale ressaltar a necessidade da existência de estudo complementares para aferir se apenas o instrumento KIDSCREEN é necessário ou, como ocorre em instrumentos direcionados à população de adultos, é necessário à inserção de um módulo complementar.

Pondera-se ainda que o Fundo das Nações Unidas para a Infância (UNICEF) estimou que há 150 milhões de crianças e adolescentes com deficiência, números considerados expressivos. Mesmo assim, o presente estudo mostra que o instrumento KIDSCREEN foi pouco utilizado em pesquisas com crianças e adolescentes com algum tipo de deficiência, perfazendo uma lacuna na literatura que merece atenção acadêmica, sendo necessário aferir se este instrumento de fato se aplica a este público específico ou se seria necessário à elaboração de um novo instrumento 
com este fim.

No grupo Violência há apenas três trabalhos referentes a essa temática. A escassez de trabalhos com este fim é encarada como justificável pelo fato da criança se deparar com uma dificuldade em dialogar sobre as agressões que sofreu, bem como o fato de que muitas vezes os adultos próximos às crianças que passaram por uma agressão não permitirem sua participação nestas pesquisas, o que limita a atuação do pesquisador em diagnosticar a qualidade de vida desse grupo (AZAMBUJA, 2006).

No que tange ao continente de vínculo do primeiro autor, ressalta-se que o instrumento KIDSCREEN foi utilizado por pesquisadores de cinco continentes, conforme os resultados apontam (tabela 3).

Tabela 3: Continente de vínculo com o primeiro autor

\begin{tabular}{ccc}
\hline CONTINENTE & $\begin{array}{c}\mathbf{N}^{\circ} \text { DE } \\
\text { PUBLICAÇÕES } \\
\text { 2001-2015 }\end{array}$ & $\begin{array}{c}\mathbf{N}^{\circ} \text { DE } \\
\text { PUBLICAÇÕES } \\
\text { 2016 }\end{array}$ \\
\hline Europa & 94 & 30 \\
América do Sul & 22 & 2 \\
América do & 9 & 2 \\
Norte & & 1 \\
Oceania & 5 & 2 \\
Äsia & 2 & \\
\hline
\end{tabular}

Fonte: Autoria própria (2016)

A utilização do instrumento KIDSCREEN por pesquisadores do continente Europeu se sobressaiu em relação aos demais continentes, provavelmente em função de que o seu desenvolvimento ocorreu em 13 países desta região7, essa ascensão perdura até o ano de 2016, sendo o continente com maior concentração de pesquisas. No entanto, como o KIDSCREEN teve um desenvolvimento transcultural (GASPAR; MATOS, 2008), é pertinente a sua utilização em outras localidades.

Segundo Fleck (2000), a maioria dos instrumentos que avaliam a qualidade de vida são desenvolvidos na Europa e nos Estados Unidos. Desta forma, a baixa utilização do KIDSCREEN por pesquisadores da América do Norte (11) justifica-se em virtude da preferência por instrumentos locais de avaliação da qualidade de vida de crianças no público infanto-juvenil.

Chama a atenção a pouca utilização do instrumento KIDSCREEN por pesquisadores do continente da Oceania e a inexistência de ocorrências com pesquisadores africanos. Ainda que a Oceania trate-se de um continente menor em relação aos demais, a justificativa para estes cenários pode estar ligada ao panorama encontrado na Ásia (4), em que também o número de pesquisadores desta localidade que utilizaram o KIDSCREEN foi bastante pequeno. Na Ásia, segundo Dey, Landolt e Mohler-Kuo (2012), a temática da avaliação da qualidade de vida em crianças é um tema novo, que teve seu início em 2012, o que justifica a baixa utilização do instrumento KIDSCREEN por pesquisadores deste local.

Em relação à nacionalidade do primeiro autor (tabela 4) observa-se que, tal qual o exposto anteriormente, a aplicação do instrumento KIDSCREEN ocorreu em maior frequência por autores da Europa e da América do Sul. 
Tabela 4: Ranking dos 11 países do primeiro autor com mais publicações sobre a utilização do KIDSCREEN

\begin{tabular}{ccccc}
\hline PAis & $\begin{array}{c}\% \\
\mathbf{2 0 0 1 - 2 0 1 6}\end{array}$ & $\begin{array}{c}\mathbf{N}^{\circ} \mathbf{D E} \\
\text { PUBLICAÇÕES } \\
\mathbf{2 0 0 1 - 2 0 1 5}\end{array}$ & $\begin{array}{c}\mathbf{N}^{\circ} \mathbf{D E} \\
\text { PUBLICAÇÕES } \\
\mathbf{2 0 1 6}\end{array}$ & TOTAL \\
\hline Alemanha & $10,6 \%$ & 14 & 4 & 18 \\
Espanha & $10,1 \%$ & 16 & 1 & 17 \\
Holanda & $8,9 \%$ & 13 & 2 & 15 \\
Portugal & $7 \%$ & 8 & 4 & 12 \\
Reino Unido & $6,5 \%$ & 9 & 2 & 11 \\
França & $6 \%$ & 6 & 4 & 10 \\
Chile & $4,7 \%$ & 6 & 2 & 8 \\
USA & $4,7 \%$ & 6 & 2 & 8 \\
Suiça & $4 \%$ & 6 & 1 & 7 \\
Brasil & $3,5 \%$ & 6 & - & 6 \\
Austrália & $3,5 \%$ & 5 & 1 & 6 \\
\hline
\end{tabular}

Fonte: autoria própria (2016)

Na tabela 4 constam os 11 países com maior número de publicações. A Alemanha atingiu 10,6\% das 169 publicações selecionadas para a presente pesquisa, passando na frente da Espanha no ano de 2016, o ano o qual obteve quatro publicações contra uma publicação da Espanha, motivo pela qual é o primeiro país ranqueado na tabela 5. Já no continente da América do Sul, pesquisadores do Brasil e do Chile totalizaram juntos $8,2 \%$ das publicações analisadas, destacando-se neste cenário.

Na América do Norte, os pesquisadores dos Estados Unidos atingiram 4,7\% das publicações, destacando-se neste continente. Já na Oceania, pesquisadores da Austrália foram os únicos no continente a utilizar os instrumentos KIDSCREEN para avaliar a qualidade de vida de crianças e adolescentes.

\section{CONCLUSÃO}

A utilização do instrumento KIDSCREEN vem crescendo nos últimos anos, por pesquisadores da área, evidenciando que o meio acadêmico tem valorizado tal temática. Este aumento quantitativo tende a contribuir com a evolução acadêmica desta área, com descobertas relevantes e inovadoras.

Vale salientar que os periódicos que obtiveram mais publicações sobre a utilização do instrumento KIDSCREEN em crianças e adolescente foram os específicos da área de qualidade de vida. Em relação à utilização das três versões do instrumento KIDSCREEN, naturalmente o KIDSCREEN-52, considerado progenitor, foi o mais utilizado pelos pesquisadores, seguido pelo KIDSCREEN-27.

Ressalta-se ainda que os instrumentos KIDSCREEN são usados em diversos grupos, de populações gerais e específicas, o que sugere sua eficiência em ambos os casos. Ainda assim, 
pesquisas direcionadas às crianças com deficiência com o instrumento KIDSCREEN ainda são escassas, o que sugere a necessidade de investigação em relação a necessidade da confecção de um módulo adicional direcionado a este público.

Por fim, conclui-se que o presente estudo cumpriu com o objetivo de analisar as publicações que avaliassem a qualidade de vida no público infanto-juvenil por meio do instrumento KIDSCREEN, contribuindo para que os pesquisadores tenham mais informações válidas em relação ao referido instrumento.

\section{REFERÊNCIAS}

AZAMBUJA, Maria Regina Fay. Violência sexual intrafamiliar: é possível proteger a crianças? Revista Virtual Texto e Contexto, v. 5, n. 5, p. 1-19, nov. 2006.

CORREIA, Fernanda Ribeiro; DE CARLO, Marysia Mara Rodrigues Prado. Avaliação de qualidade de vida no contexto dos cuidados paliativos: revisão integrativa de literatura. Revista Latino-Americano de Enfermagem, v. 20, n. 2, p. 1-10, mar./abr, 2012.

DEY, Michelle; LANDOLT, Markus. A.; MOHLER-KUO, Meichun. Health-related quality of life among children with mental health problems: a population-based approach. Health Quality of Life, v. 10, n. 1, p. 73, jun. 2012.

FLECK, Marcelo de Pio Almeida. O instrumento de avaliação de qualidade de vida da Organização Mundial da Saúde (WHOQOL-100): características e perspectivas. Ciências da Saúde Coletiva. Rio de Janeiro, v. 5, n. 1, p. 33-38, 2000

FLECK, M. P. A. Problemas conceituais em qualidade de vida. In: FLECK, M. P. A. et al. (Org.). A avaliação de qualidade de vida: guia para profissionais da saúde. Porto Alegre: Artmed, 2008.

GASPAR, Tania; MATOS, Margarida Gaspar; LEAL, Isabell. Qualidade de vida e bem-estar em crianças e adolescentes. Revista Brasileira de Terapias Cognitivas, Rio de Janeiro, v. 2, n. 2, p. 47-60, dez. 2006.

GASPAR, T; MATOS, M. G. Qualidade de vida em crianças e adolescentes: versão portuguesa dos instrumentos KIDSCREEN 52. Lisboa: Aventura Social e Saúde, 2008.

GIL, Antônio Carlos. Como elaborar projetos de pesquisa. 4. ed. São Paulo: Atlas, 2002.

GRÁCIO, Maria Claudia Canbrini; OLIVEIRA, Ely Francina Tannuri de. A inserção e o impacto internacional da pesquisa brasileira em 'estudos métricos': uma análise na base Scopus. Tendências da Pesquisa Brasileira em Ciência da Informação, v. 5, n. 1, p. 1-19, 2012.

HIDALGO-RAMUSSEN, Carlos Alejandro; RAJMIL, Luis; ESPINOZA, Rosa Montaño. Adaptación transcultural del cuestionario KIDSCREEN para medir calidad de vida relacionada con la salud en población mexicana de 8 a 18 años. Ciência e Saúde Coletiva, Rio de Janeiro, v. 19, n. 7, p. 22152224, jun./out. 2014.

JERVAEUS, A.; KOTTORP, A.; WETTERGREN, L. Psychometric properties of KIDSCREEN-27 among childhood cancer survivors and age matched peers: a rasch analysis. Health Quality of Life Outcomes, v. 13, n. 1, p. 11-96, jun. 2013. 
JIROJANAKUL, Pragai; SKEVINGTON, Suzanne. Developing a quality of life measure for children aged 5-8 years. British Journal of Health Psychology, v. 5, n. 3, p. 299-321, set. 2000.

LANDEIRO, Graziela Macedo Bastos et al. Revisão sistemática dos estudos sobre qualidade de vida indexados na base de dados Scielo. Ciências e Saúde Coletiva, Rio de Janeiro, v. 16, n. 10, p. 4257-4266, 2011.

RAVENS-SIEBER, U. et al. The European KIDSCREEN approach to measure quality of life and well-being in children: development, current application, and future advances. Quality of Life Research, Oxford, v. 23, n. 3, p. 791-803, abr. 2014.

SEIDL, Eliane Maria Fleury; ZANNON, Célia Maria Lana da Costa. Qualidade de vida e saúde: aspectos metodológicos e conceituais. Caderno de Saúde Pública, Rio de Janeiro, v. 20, n. 2, p. 580-588, mar./abr, 2004.

SOARES, A. H. R. et al. Qualidade de vida de crianças e adolescentes: uma revisão bibliográfica. Ciência e Saúde Coletiva, Rio de Janeiro, v. 16, n.7 p. 3197-3206, jul. 2011.

Recebido em: 26-04-2018

Aceito em: 01-07-2019 